\title{
The psychogeriatrician's view: management of chronic disability in the community
}

\author{
D G Wilkinson
}

\begin{abstract}
Psychiatric services for elderly people are based on principles which are relevant to the management of chronic disorders such as Parkinson's disease. Psychological and biological factors are closely intertwined in dementia, as they are in PD. Although medical diagnosis is important the emphasis is on continuing support. The aim is to provide services within the community where they are needed, and to acknowledge the importance of carers and family for assessment and management.
\end{abstract}

It may seem that psychiatry has little to offer neurology in the management of chronic neurological disability. However, psychiatrists have a long association with neurological disability, often assuming responsibility for certain groups of patients and being involved in the treatment of the psychiatric sequelae in others. The psychiatric morbidity may be associated with the difficulties in adjusting to the illness, as a result of altered behaviour due to brain damage, or as a result of treatment of the underlying condition.

In recent years psychogeriatricians have taken on the mantle of organic psychiatry and have developed wide experience in the management of dementia. They are involved in the whole range of psychiatric illness and place great emphasis on the curative aspect of the work, particularly the diagnosis and treatment of depression.

There are few chronic irreversible diseases which do not precipitate depression in some of their sufferers and acknowledgement of that, and sensitivity to the patients' feelings are an important part of the response to therapy. If clinicians want to be truly involved in the management of chronic disability they will have to heed the patients' experience of their illness. There can be little benefit in telling the patient he is technically better if he does not feel better.

Such assertions undermine the patients' confidence in the doctor who is then seen to be unhelpful with no understanding of their "real" problems. The patient may then be reluctant to express his or her feelings and problems for fear that they will be dismissed, and compliance with treatment may then suffer. In Parkinson's disease, for example, the loss of tremor and decreased tone are important therapeutic goals, but if they are achieved at the expense of disturbed nights which increase carer strain with consequent irritability, the patient may not benefit. A patient may be seen as difficult when he refuses a catheter for urinary incontinence despite the obvious burden to his wife, but it may be that no one has asked about his sexual activity and addressed his fears of how catheterisation may take away his last vestige of self-esteem.

Lists of psychiatric symptoms in Parkinson's disease are commonplace, with the prevalence of depression varying from $30-70 \% .^{1-4}$ However, a recent article on treatment ${ }^{5}$ failed to mention depression at all.

This seems to be a peculiar blind spot as the treatment of depressive symptoms offers great scope for improvement, and there are those who advocate electroconvulsive treatment (ECT) as the treatment of choice for those with depression ${ }^{67}$ and even for non depressed ${ }^{8}$ Parkinsonian patients. I have no doubt from personal experience that ECT improves the motor symptoms in depressed Parkinson's disease sufferers, but actual or incipient dementia can prevent improvement and produce increasing confusion during treatment.

The problem seems to be that we often regard depression and psychological well being as being separate from the underlying disability. Reynolds ${ }^{9}$ feels psychiatrists should stop arguing about this false dichotomy and neurologists might usefully spend more time studying the influence of psychological and social factors on brain function and on their patients' functional disabilities. In the area of chronic disability the difference between neurology and psychogeriatrics seems to have less to do with content and more to do with style of delivery. Neurologists are a scarce resource and diagnosis has always been their raison d'être. By contrast the psychogeriatrician, whilst still a diagnostician, has necessarily become expert at interdisciplinary working in the community.

We must retain our diagnostic role and it is essential that every referral to our service is seen by a member of the medical staff. The value of this is borne out by experience. Extreme cases may be rare, but nevertheless they shape our practice. One case illustrates the need to rely on clinical diagnosis and listen to carers. Some years ago I was repeatedly asked by a professorial unit to take over the care of a 57 year old diabetic man for long term care of his presenile dementia. He had a history of boxing, probably heavy drinking and depression, but had had a rapid mental decline into
Moorgreen Hospita D G Wilkinson 
Table Southampton psychogeriatric service

\begin{tabular}{lrrr}
\hline & 1983 & 1986 & 1989 \\
New cases & 725 & 833 & 817 \\
Admissions & 842 & 930 & 1032 \\
Homes visits by doctors & 5690 & 7191 & 9278 \\
Home vists by community & & & 13150 \\
$\quad$ psychogeriatric nurses & & & \\
\hline
\end{tabular}

mutism, had become totally dependent and was incontinent of urine. Numerous investigations had been carried out and a neurological assessment had been obtained. CT and EEG were said to confirm the diagnosis of dementiaprobably Pick's or Alzheimer's disease, but the rapid course meant Creutzfeld-Jacob disease could not be excluded. His history and that of his family suggested depression as the most likely diagnosis, and that he was not demented. He needed intravenous fluid. Twenty ECT treatments were given at my request. The patient, however, was still mute and now doubly incontinent, so I agreed to take over his case. He was admitted, given 10 more ECTs, put on lithium and carbamazepine as well as his antidepressants. For the past three years he has been living at home with his family with no signs of cognitive impairment, and no evidence of depression, except during one brief relapse after he stopped taking his lithium. "So treat the patient not the tests" is an axiom we should never forget.

This good outcome has much to do with accurate diagnosis but also depended on close follow up at home, as the patient refused to attend hospitals, perhaps understandably. Without home follow up his relapse might not have been dealt with early and he might not have complied subsequently with medication and consequently required further prolonged admissions. One of the findings of our three year follow up of patients given ECT was that assiduous follow up was an important factor in good outcomes. ${ }^{10}$

As this case illustrates, outpatient clinics for elderly disabled patients are not cost effective, particularly from a wide catchment area with problems of cost and availability of ambulance transport. By more or less dispensing with outpatient clinics, except for routine lithium clinics, we can aim to assess all patients at the point of referral, whether at home or in hospital within 24 hours. The table shows rates of referral of new cases to a psychogeriatric unit; it does not include re-referrals, even if the referral was for a new illness episode. Home visits are carried out mostly by the three consultants, but the junior doctors do undertake small caseloads and their visits are included. If we include the 12 community psychiatric nurses' visits too, home visits amount to 22000 per year. This pattern of service pays enormous dividends in terms of support for patients and carers, information gathering which puts the illness in context and the rapid treatment of treatable illness and prevention of inappropriate admissions to hospital or residential care.

The psychogeriatric community nurses are a growing and vital part of the programme of community support. Their role is to become a key worker for a patient, predominantly those with organic illness, and is as applicable to patients with Parkinson's disease, as those with dementia. They form a crucial link between patient, family and hospital, serving to monitor progress with medication and adjusting doses within present guidelines. They can ensure domiciliary services like home care, day care and any financial benefits are obtained, take charge of arranging respite admissions to hospital and liaise with social services homes if more appropriate.

They counsel and support carers both informally and through carers groups, and supply the "hot-line" to carers which is essential if we are to limit the strain and uncertainty. They can offer immediate help in terms of medical review, admission and re-assessment of services, calling in an occupational therapist or psychologist, if required. They liaise closely with our psychogeriatric sitting service, which offers carers up to 15 hours a week of sitting time $^{11}$ and our day hospitals which can offer only one or two days attendance a week at present.

Day hospital care is an important means of monitoring change in chronic disability. The function of a psychogeriatric day hospital stretches from the relatively short focused attendance for the functionally ill to permanent attendance until residential care is necessary for some patients with dementia. Long term attendance allows the development of prosthetic bonds ${ }^{12}$ which often can be sufficient to maintain the patient and carer at home in the community. The problem of ambulance transport to day hospitals is ever present, and patients from the outer parts of our catchment area are precluded from attending our day hospital through lack of transport. I started a travelling day hospital in 1986 which entailed a group of nurses and an occupational therapist driving their own mini-bus to a different location in the more distant parts of our catchment area each day.

They pick up about 15 patients per day who are predominantly severely demented patients being cared for at home; (patients living locally can make their own way). This has proved successful, but hard work for staff. Carers are delighted at the chance of respite previously denied to them, they are confident in the care because the staff themselves call to collect and return the patient, so direct communication is possible. The local non-hospital venues which are mostly little used day areas in sheltered housing units, offer a relaxed environment which facilitates assessment and rehabilitation and outweighs the lack of specialist equipment. They have been able to care for profoundly demented patients and have also been able to provide imaginative integration of chronically functionally ill patients using individual and group therapies.

We are, however, facing an increasing burden particularly from the organic disorders; we see around 400 cases of dementia each year, as well as those that are functionally ill. That means we see about eight cases of dementia that are totally new to our service each week. Clearly 
the vast majority of these patients are not, and cannot be, extensively investigated. Virtually none will have CT brain scans, because until recently our service had been denied access unless the scan was authorised by a neurologist.

It has now become easier to arrange scans, but if we had carte blanche we would certainly not scan them all, as I do not share with others the touching faith in CT scanning and its ability to aid diagnosis in clinically demented patients. Although it may be heresy to say so, the management of these patients is unlikely to be influenced by the result, whether it shows multi-infarct or frontal dementia, gross atrophy or a normal scan. Even signs of normal pressure hydrocephalus seem to result in very little surgical intervention these days. Dementia is a clinical diagnosis and no amount of investigation will alter this fact. The precise cause will matter very little in the day to day management of the patient unless a significant treatment for any of the dementing processes emerges. What is far more important is ensuring the carer has access to, and help with all the relief, aids and allowances they can obtain. Also that the carer has a hot line that brings an immediate response and, when the chips are down, same day admission for respite. It is important that the community psychiatric nurse, day hospital, occupational therapy assessment, day centre, sitting service, carers groups, home care and any other relevant support are introduced with speed and sensitivity.

That is the way to reduce disability. By following the patient one can be aware of change in the patient. If the carer starts calling for help when the patient appears no worse, one can be aware of carer strain and probable depression which needs urgent treatment or support rather than being brushed off with comments that there appears to be no deterioration in the patient or by tinkering with his medication-as one might do if seeing the patient in isolation in a clinic.

It would be helpful if greater liaison and awareness of neurological and psychogeriatric services were possible. At the moment things are rather haphazard, in that either service might become involved depending on which door the patient knocks on first. What I would like is that whichever door it is, the patient is able to enjoy the benefit of either service. What I do not want is to get referrals of patients with dementia from general practitioners who say "Mrs A has been stoically coping with her husband who was investigated at the neurological centre two years ago and diagnosed as having Alzheimer's disease. She is now finding it difficult to cope, could you assess?" She should have been having support two years ago and might not be collapsing now if she had. I never object to seeing people early in their dementia. I am distressed by picking up the pieces of an already shattered caring network which no amount of psychogeratric superglue can fix.

Although I have been mainly addressing dementia, Parkinson's disease is probably the illness which epitomises the need for liaison. I find the management of patients with Parkinson's disease who are referred to me very difficult for a variety of reasons, but particularly because of the complex nature of the interaction between functional disturbances and organic illness.

In two summer months we saw 34 patients with Parkinsonian symptoms including 11 new cases and 23 follow up visits and there were six admissions to the psychiogeriatric unit. If we extrapolate we might see about 60 new cases of PD or Parkinsonism in a year who generate perhaps some 300 visits, including follow ups.

Men and women were equally represented, but although the average age of the women was slightly higher it was interesting that less than $20 \%$ of the men were over 80 , whereas half of the women were. It is my impression that men with Parkinson's disease cause much more work in terms of behavioural and psychological problems, and whether they have a greater mortality as a result, or whether the figures simply reflect the expected demography, is uncertain.

If we look at the cause of these symptoms about $65 \%$ had idiopathic Parkinson's disease (14 male and 8 female). There were more women (10) in the iatrogenic group than men (2), which agrees with Ayd's work suggesting drug induced Parkinson's disease is correlated with older age and female gender. ${ }^{13}$

The 12 patients with drug induced symptoms could be divided into three groups. Five were chronic schizophrenics on long term neuroleptic drugs, one of whom also had buccolingual dsykinesia. Three had dementia with symptoms attributable to long term neuroleptics given for behavioural or psychotic symptoms-these patients are not routinely given anticholinergenics. Four had depression and all four were on flupenthixol $0.5 \mathrm{mg}$ daily as well a tricyclic antidepressent and three were also on lithium. There may be a synergism between flupenthixol and lithium in inducing extrapyramidal signs, as has been noted with higher doses of haloperidol and some depot neuroleptics and lithium.

Half of the new cases were referred because of the visual hallucinations often regarded as secondary to medication for their Parkinson's disease. Perry ${ }^{14}$ suggests that Lewy body type dementia is associated with visual hallucinations and that some patients with Parkinson's disease may have hallucinations as a result of this rather than as a complication of their treatment. This agrees with my experience. Half of the patients were referred because of depression, $35 \%$ because of dementia, and $15 \%$ because of schizophrenia.

As I have already described, our management of these patients is often supportive. Those on neuroleptic drugs need constant review to keep the balance of medication effective and many are a constant reminder of how fallible and impotent we are as specialists in the relief of certain forms of intractable distress. What is clear is that we need a more eclectic view of this and many other diseases.

In old age psychiatry, the advent of new imaging techniques seems to show that depres- 
$\operatorname{sion}^{15-17}$ is associated with changes in structure and function of the brain as does schizophrenia. ${ }^{18}$ As ever in psychiatry we seem to have completed another circle. The seminal work of Sir Martin Roth in the $1950 \mathrm{~s}^{19}$ identified depression and dementia as distinct entities among what had hitherto been encompassed by the term senile psychosis. This distinction seems less obvious and secure now. Consequently, we have to work together, and learn from each other. Accurate lesion finding and diagnosis may be of crucial importance for psychogeriatrics if new treatments are found for any of the disease processes so far crudely incorporated under the Alzheimer's disease label. This will be especially true if these treatments have significant side effects. Neurologists may wish to spend more time considering the after-care and rehabilitation role with patients, an area as rewarding as the quest for the lesion.

1 Celesia GG, Wanamaker WM. Psychiatric disturbances in Parkinson's disease. Diseases of the nervous system 1972;33:577-83.

2 Mindham RHS. Psychiatric symptoms in parkinsonism. Neurol Neurosurg Psychiatry 1979;33:188-91.

3 Mayneux R, Stern Y, Rosen J, Leventhal J. Depression intellectual impairment, and Parkinson's disease. Neurology 1981;31:645-50.

4 Warburton JW. Depressive symptoms in Parkinson patients referred for thalamotomy. J Neurol Neurosurg Psychiatry 1967;30:368-70.

5 Bakheit AMO. Drug treatment of Parkinson's disease, Hospital Update 1990;16(6):497-504.

6 Lebonsohn ZM, Jenkins RB. Improvement of parkinsonism in depressed patients treated with ECT. Am J Psychiatry 1975;132:283, 285.

7 Asnis G. Parkinsons disease, depression and E.C.T.; a review and case study. Am J Psychiatry 1977;134:191-5.

8 Balldin J, Granerus AK, Lindstedt G, Modigh K, Wallinder J. Predictors of improvement after electroconvulsive therapy in parkinsonian patients with on-off symptoms. therapy in parkinsonian patients with on-off sy
Journal of Neural Transmission 1981;52:199-211

9 Reynolds EH. Structure and function in neurology and psychiatry. Br J Psychiatry 1990;157:481-90.

10 Godber C, Rosenvinge HP, Wilkinson DG, Smithies J. Depression in old age: prognosis after ECT International Journal of Geriatric Psychiatry 1987;2:19-24.

11 Rosenvinge H, Guigon J, Dawson J. Sitting Service for the elderly confused: Part of an integrated programme of management. Health Trends 1986;18:47.

12 Arie T. Day care in geriatric psychiatry. Gerontologica Clinicia 1975;17:31-9.

13 Ayd FJ. A survey of drug induced extrapyramidal reactions. J Am Med Assoc 1961;175:1054-60.

14 Perry R, Irving D, Perry E. Visual hallucinations as the presenting symptom of dementia a type of Lewy body disease? International Journal of Geriatric Psychiatry 1990;5:275-6.

15 Jacoby RJ, Levy R. Computed tomography in the elderly: 3 , affective disorder. Br J Psychiatry 1980;136:270-5.

16 Dolan J, Calloway SP, Mann AH. Cerebral ventricular size in depressed subjects. Psychological Medicine 1985; 15:873-8.

17 Ames D, Doolan R, Mann A. The distinction between depression and dementia in the very old. International Journal of Geriatric Psychiatry 1990;51:193-8.

18 Johnstone EC, Crow TJ, MacMillan JF, Owens DG, Bydder GM, Steiner RE. A magnetic resonance study of early schizophrenia. J Neurol Neurosurg Psychiatry 1986; 49:136-9.

19 Roth $M$. The natural history of mental disorder in old age. Journal of Mental Science 1955;101:281-301. 